\title{
Midwifery Core Competency Assessment Tool: A Systematic Review Protocol
}

\author{
Shenmei Li, Yunyun Dai", Yangguang Chen, Yan Gao
}

Department of Nursing, Guilin Medical University, Guilin, China

Email address:

529458241@qq.com (Yunyun Dai)

${ }^{*}$ Corresponding author

\section{To cite this article:}

Shenmei Li, Yunyun Dai, Yangguang Chen, Yan Gao. Midwifery Core Competency Assessment Tool: A Systematic Review Protocol. American Journal of Clinical and Experimental Medicine. Vol. 9, No. 1, 2021, pp. 13-16. doi: 10.11648/j.ajcem.20210901.13

Received: January 20, 2021; Accepted: March 3, 2021; Published: March 9, 2021

\begin{abstract}
Background: in recent years, the capacity building of midwives has attracted the attention of all countries in the world, especially in countries with a lack of midwifery human resources. Improving the capacity of midwives is an important strategy to ensure the health of mothers and infants. Professional associations in many countries have issued standards for midwife competency, which are basically similar to the core competency module proposed by ICM, but some countries have made localization adjustments according to their own characteristics. In August 2019 a further update was made to correct an error in competency of the English version, the French and Spanish versions by ICM. In order to further standardize the evaluation tools of midwives' core competence, it is urgent to evaluate them. Methods/Design: We will conduct a systematic review and utility critique of instruments used to measure effectiveness of core competence assessment tools for midwives at different levels. The databases Medical Literature Analysis and Retrieval System Online (MEDLINE), Cumulative Index to midwife competency and Web of Knowledge will be searched from inception until end November 2020. Search strategies will include the key words; midwife, core competency, questionnaires, instruments, delphi technique, index system, reliability, validity. We will contact experts in the field of measuring core competency and scrutinise all secondary references. A reviewer will apply an inclusion criteria scale to all titles and abstracts. A second reviewer will apply the inclusion criteria scale to a random 10\% selection. Two reviewers will independently evaluate the methodological rigour of the testing of the instruments using the Consensus-based Standards for the selection of core competency checklist. We will present a narrative synthesis on the utility of all instruments and make recommendations for instrument selection in practice. Discussion: This systematic review of the utility of instruments to measure the core competence assessment tools for midwives at different levels. It will aid managers, educator and policy makers to select an instrument fit for purpose. Importantly, appropriate instrument selection will provide a mechanism for effective training of midwives' core competency ability in different levels.
\end{abstract}

Keywords: Core Competency, Midwifery, Assessment Tool, Systematic Review, Protocol

\section{Introduction}

The core competence of midwives is defined as "in midwifery practice and education, midwives can be competent for the combination of skilled and accurate knowledge, professional behavior and specific skills displayed in the post". The core competence of midwives is an important comprehensive index to measure the competence of midwives. International Confederation of Midwives, ICM established "midwife core competency 2002 edition, at present, the latest version of core competency standard is essential competencies for midwifery practice 2019 [1]. IN 1978, American College of Nurse Midwives, ACNM has set up different competency standards for different levels of midwives, including the core competency of basic midwifery and the competency of midwifery education at master level [2]. In 2005, Canadian Midwifery Regulators Council, CMRC issued the "Canadian core competency standard for midwives", which was revised in 2010 [3, 4]. Australian Nursing and Midwifery Accreditation Council, ANMAC established the "national midwife competency standard" in 2004 [5, 6]. British Nursing and Midwifery Council NMC issued the "core competency standard for registered midwives" in September 2010 [7, 8]. In 2011, Wang Dehui developed China's "midwife core 
competency table" [9].

As one of the three pillars of developing midwifery specialty and providing high-quality maternal and infant health services, midwifery education plays an extremely important role. Assessment of clinical practice is a core component of midwifery education. Clinical assessment is challenging and affected by a number of factors [14]. The learning goals and evaluation strategies of competency-based midwifery programs must be explicit and well-defined. In the US, didactic learning is evaluated through a standardized certification examination, but standardized clinical competence evaluation is lacking [15]. According to the state of midwifery report 2014, investment in midwifery education can receive up to 16 times of investment return [10]. In recent years, the capacity building of midwives has attracted the attention of all countries in the world, especially in countries with a lack of midwifery human resources. Improving the capacity of midwives is an important strategy to ensure the health of mothers and infants. Professional associations in many countries have issued standards for midwife competency, which are basically similar to the core competency module proposed by ICM, but some countries have made localization adjustments according to their own characteristics. In August 2019 a further update was made to correct an error in competency of the English version, the French and Spanish versions by ICM [11]. In order to further standardize the evaluation tools of midwives' core competence, it is urgent to evaluate them [13].

Therefore, on the one hand, this study attempts to explore the main content of the midwife core competency evaluation tool from the perspective of system evaluation, in order to provide reference for the revision of the midwife core competency standard. on the other hand, the purpose of this study is to find the best recommended guidance for the evaluation tools of midwife core competence, and to provide a scientific method for the evaluation of the results of clinical midwife educators and midwives core competence.

\section{Methods and Design}

\subsection{Research Question}

What are the best assessment tools for core competencies of midwives at different levels?

What are the reliability and validity of these assessment tools?

Whether these assessment tools are also suitable for the core competency guide for midwives revised by ICM in 2019?

\subsection{Aims}

To find out the best evaluation tool for different levels of midwives' core competence.

To evaluate the reliability and validity of these evaluation tools.

To assess the consistency of these assessment tools with the revised midwife core competency guidelines of the International Federation of midwives 2019.

\subsection{Keywords}

Identify key words to search articles related to midwife core competency assessment tool: midwife; core competency; competency assessment tool.

\subsection{Types of Outcomes}

This review will consider studies that investigated the core competency index and content of midwives (junior and senior) and midwives students were involved, and the best assessment tool for core competence of midwives at different levels was found, rather than involving midwives training program or midwife nursing education literature analysis.

\subsection{Inclusion Criteria}

Used a qualitative method, quantitative method, or mixed method of data collection and analysis.

Study must be the primary research studies.

Studies must be published in English or Chinese (because we have access to Chinese investigators but not other languages, and we do not have funds to access interpreter services).

It involves the core competency indicators and contents of midwives (junior, senior) and midwives.

These documents were published from January 2002 to December 2020. These documents were published from January 2002 to December 2020.

\subsection{Exclusion Criteria}

Studies are systematic reviews, opinion pieces, editorials, commends or letters should be excluded.

Study does not include the core competency indicators which should be excluded.

Non midwives and non midwives students Study should be excluded.

Other contents related to midwife training program or midwife nursing education literature analysis.

Use one-dimensional assessment tools.

Lack of reliability and validity of evaluation tools

\subsection{Search Strategy}

A three-step search strategy will be utilized in this review. An initial limited search of Medline was undertaken including the terms derived from the "PICO" in Table 1 with the aim of identifying indexed terms from relevant titles and abstracts to refine the search strategy. A comprehensive search using all identified keywords and indexed terms will then be undertaken across all included databases (Medline, EMBASE, PsycINFO, CINAHL, Chinese CNKI) from 2002 to today. Thirdly, a hand-search of the reference lists of all relevant articles will be conducted to identify additional studies that may not have been identified through the databases searches. The search for grey literature will include: ProQuest Dissertations and Theses, relevant organizations' websites. 
Table 1. PICO terms used to derive search strategy.

\begin{tabular}{|c|c|c|c|}
\hline Population & Intervention or exposure & Comparison & Outcome \\
\hline $\begin{array}{l}\text { Midwifery; } \\
\text { Junior midwife; } \\
\text { Senior midwife; } \\
\text { Midwifery students; } \\
\text { Midwifery graduates; } \\
\text { Advanced midwifery practitioner; } \\
\text { Midwifery clinical specialist; } \\
\text { Midwifery educator; }\end{array}$ & $\begin{array}{l}\text { Behavioral problems; } \\
\text { Professional assessment; } \\
\text { Midwife attitude; } \\
\text { Core competency; } \\
\text { Assessment tools; } \\
\text { Clinical competence; } \\
\text { Professional competence; } \\
\text { Midwifery Care; } \\
\text { Midwives' skills; } \\
\text { Delphi Technique index system }\end{array}$ & No & $\begin{array}{l}\text { Purpose } \\
\text { Sample size } \\
\text { Applicable people } \\
\text { Questionnaire recovery rate } \\
\text { Reliability } \\
\text { Validity } \\
\text { Cronbach's } \alpha \\
\text { Evaluating indicator } \\
\text { Educational Impact } \\
\text { Acceptability }\end{array}$ \\
\hline
\end{tabular}

\subsection{Data Management}

Search results will be exported from each databases into an Endnote Library where duplicates will be identified and removed. From Endnote, references will be exported to the systematic review management software program Covidence. Title and abstract screening, full text screening and quality appraisal will be managed in Coindence. An MS Excel worksheet will be used to store and manage extracted data.

\subsection{Assessment of Methodological Quality}

Paper selected for retrieval will be assessed by two independent reviews for methodological validity prior to inclusion in the review. Standardized critical appraisal instruments appropriate to the study design from the JBI Reviewer's Manual will be used and the procedures for critical appraisal outline in the manual will be followed. The JBI Reviewer's Manual includes tools to assess various quantitative and qualitative study designs. Any disagreement that arise between the reviewers will be resolved with a third reviewer.

\section{Data Extraction}

Data will be extracted from papers selected for inclusion using customized versions of the data extraction tools for qualitative, quantitative studies from the JBI Reviewers Manual. Data extraction will be completed independently by two reviewers. A third reviewer will independently perform data extraction in the event that there are discrepancies in the data extracted by the initial two reviewers.

\section{Data Synthesis}

We will use a mixed methods approach to synthesize qualitative and quantitative data. In the first phase, we will extract qualitative data from qualitative, quantitative, and mixed methods studies. We will use thematic analysis to identify relevant themes related to assessment tools for core competence of midwives at different levels.

\section{Discussion}

At present, the core competency standards of midwives are different in different countries and different regions of the same country. The reliability, validity and indicators of each evaluation tool are different, which is not conducive for midwife educators and clinical practitioners to refer to the most authoritative standards, thus affecting their training of the best midwives. Improving and sustaining the capacity of midwives continues to challenge practitioners and policy makers [12]. This systematic review of the utility of instruments to measure the core competence assessment tools for midwives at different levels. It will aid managers, educator and policy makers to select an instrument fit for purpose. Importantly, appropriate instrument selection will provide a mechanism for effective training of midwives' core competency ability in different levels. In addition, it can save the time cost, human cost and financial cost of midwife educators and clinical practitioners in various countries and also provide evidence support for them to choose the best midwife core competency evaluation criteria.

In short, to find the best midwife core competency evaluation criteria is conducive to midwife educators and clinical practitioners to better train midwives to adapt to the future social development.

\section{Conclusion}

The core competence of midwives is of great significance to their own career development, and is affected by age, education background, cognition, psychological state, marital status and other factors. Therefore, it is very necessary and urgent to explore the influencing factors of midwives' core competency and develop a practical core competency evaluation scale. Only by establishing a practical evaluation index, can we better cultivate excellent midwives and provide the basis for midwives' education reform, so as to promote the better and faster development of midwives' Education. We believe that this systematic review is timely and will make a valuable contribution to fill an existing research gap.

\section{Conflicts of Interest}

The authors declare that they have no competing interests.

\section{Acknowledgements}

We would like to thank the Guangxi Education Hall for financing the research. 
(Research Fund No. 2020KY12036; Research Fund No. 2018glmc026)

\section{References}

[1] ICM. Essential for basic midwifery practice 2010 Revised $2013 \quad$ [EB/OL]. [2017-08-25]. http://internationalmidwives.org/assets/uploads/documents/cor edocuments/ICM\%20Essential\%20

Competencies\%for20Basic\%20Midwifery\%20Practice\%2020 $10, \% 20$ revsed\%202013.pdf.

[2] ACNM./core Competencies for Basic Midwifery Practice 2012 [EB/OL]. [2017-08-25]. http:/midwives.org/ACNM/files/ACNMLibraryData/UPLOA DFILENAME/0000000.

[3] CMRC. About CMRC [EB/OL] [207-08-25] $\mathrm{http}: / / \mathrm{cmrc}-\mathrm{ccosfl} \mathrm{ca} /$ about-cmr cinitiatives.

[4] CMRC. Canadian Competencies for Midwives [EB/OL] [207-08-25]

http://cmrc-ccosf.ca/sites/default/files/pdf/National-Compente ncies-ENG-rev08.pdf.

[5] ANMAC. About ANMAC [EB/OL] [207-08-25] http://www.anmac.org.au/about-anmac.

[6] Ausustrtian Nursing \& Midwifery Accreditation Council. Programs Leading to Endorsement for Scheduled Medicines for Midwives Accreditation standards2015 [S/OL] [2017-08-25].

http://www.anmac.org.au/sites/default/files/documents/Accedi taion-Standards-for-Programs-Leading-to-Endorsement-for-Sc heduled-Medi-cines-for -Midwwives-015.pdf.
[7] NMC. Our Role-What we do [EB/OL [2017-08-25] https://www.nmc.org.uk/bout-us/our-role/.

[8] NMC. Standards for competence for registered midwies $[\mathrm{EB} / \mathrm{OL}]$

[2017-0-25] https://www.nmc.org.uk/standards-o-competence-for-registere d-midwives/.

[9] WANGDe -hui, LU Hong, SUN Hong, Midwife Core Competency Scale: Reliability and validity assessment. Chinese Nursing Management 2011, 11 (12): 42-45.

[10] United Nations Population Fund (UNFPA) World Health Organization (WHO), state International Federation of midwives (ICM). 2014 Report on midwifery Path: Women's right to health, Prague: UNFPA, WHO, ICM. 2014.

[11] Essential Competencies for Midwifery Practice 2019 UPDATE. International Confederation of Midwives (Strenthenging Midwifery Globally), 2019, 10.

[12] Michelle Beattie, William Lauder, Iain Atherton (2014). Instruments to measure patient experience of health care quality in hospitals: a systematic review protocol. Systematic Reviews, ( $p p$ 4-8).

[13] Nora Suleiman-Martos, Luis Albendín-García, José L. Gómez-Urquiza, etal. (2020). Prevalence and Predictors of Burnout in Midwives: ASystematic Review and Meta-Analysis. Int. J. Environ. Res. Public Health 17, 641.

[14] Carmel Bradshaw, Judith Pettigrew, Mary Fitzpatrick (2019). Safety first: Factors affecting preceptor midwives experiences of competency assessment failure among midwifery students. midw Jul; 74: 29-35.

[15] Kate Woeber (2018). Development and implementation of a competency-based clinical evaluation tool for midwifery education. midw Jul; 62: 92-95. 